\title{
Surface polaritons of a left-handed material slab
}

\author{
R Ruppin \\ Soreq NRC, Yavne 81800, Israel
}

Received 5 January 2001, in final form 30 January 2001

\begin{abstract}
The dispersion relations of the surface polaritons of a slab made of a material which has dispersive permittivity and permeability, and is left-handed over a frequency band in the microwave range of several $\mathrm{GHz}$, are investigated. Four branches of p polarized surface polaritons and two branches of $s$ polarized ones are found to exist. The possibility of experimentally observing the surface polaritons by the attenuated total reflection method is demonstrated.
\end{abstract}

\section{Introduction}

Recently, a composite material has been produced, for which the effective permittivity and the effective permeability are both negative over a finite frequency band [1]. A medium of this type is called 'left-handed' because for an electromagnetic plane wave propagating in it, the direction of $\vec{E} \times \vec{H}$ is opposite to that of the wavevector. The peculiar electromagnetic properties of left-handed materials, such as reverse Doppler shift and Cherenkov radiation and inverse Snell effect, have been discussed theoretically a long time ago by Veselago [2]. It has been suggested that slabs of left-handed materials could be employed as an unconventional alternative to lenses, and in this context, evanescent waves play an important role [3]. Here we investigate the surface polaritons of a left-handed material slab. These polaritons are those electromagnetic normal modes of the slab, which have an evanescent behaviour both inside and outside the slab.

To create the left-handed medium, an array of wires has been used, interspersed with an array of split ring resonators [1]. The thin wire array has been shown by Pendry et al [4] to yield an effective dielectric constant of the form

$$
\varepsilon(\omega)=1-\frac{\omega_{p}^{2}}{\omega^{2}}
$$

with the plasma frequency $\omega_{p}$ in the GHz range. The split ring resonator array has been shown by Pendry et al [5] to yield an effective magnetic permeability of the form (neglecting damping for the moment)

$$
\mu(\omega)=1-\frac{F \omega^{2}}{\omega^{2}-\omega_{0}^{2}}
$$

with the resonance frequency $\omega_{0}$ in the $\mathrm{GHz}$ range. The size and spacing of the conducting elements of which the medium was composed were on a scale much smaller than the 
wavelengths in the frequency range of interest [1]. We can therefore represent the composite medium as continuous and homogeneous. Also, it has been shown that any interactions between the wires and the split ring resonators are negligible [6]. The composite medium reported in [1] was anisotropic, but it was predicted that by adding elements to increase the symmetry an isotropic left-handed medium can be achieved. We will therefore perform the analysis for a slab made of a homogeneous isotropic material, having permittivity and permeability of the form (1) and (2), respectively.

The dispersion relations of the $p$ polarized and $s$ polarized surface polaritons of the slab are derived in Section 2. The excitation of the surface polaritons by the method of attenuated total reflection (ATR) is discussed in Section 3.

\section{Surface polaritons}

We derive the surface polariton dispersion relations for a slab of thickness $d$. The slab, which is characterized by a dielectric constant of the form (1), and a magnetic permeability of the form (2), occupies the region $0<x<d$. The media at $x<0$ and $x>d$ have a dielectric constant $\varepsilon_{m}$ and a magnetic permeability $\mu_{m}$, both of which are assumed to be frequency independent.

\section{1. p polarization}

In the region $x<0$ the electromagnetic fields have the form

$$
\begin{aligned}
\vec{H}^{(1)} & =\hat{a}_{y} A \mathrm{e}^{k_{0} x} \mathrm{e}^{\mathrm{i} h z} \\
\vec{E}^{(1)} & =\frac{c}{\omega \varepsilon_{m}} A\left(h \hat{a}_{x}+\mathrm{i} k_{0} \hat{a}_{z}\right) \mathrm{e}^{k_{0} x} \mathrm{e}^{\mathrm{i} h z} .
\end{aligned}
$$

Here $\hat{a}_{x}, \hat{a}_{y}, \hat{a}_{z}$ are unit vectors in the $x, y$ and $z$ directions, respectively. The relation between the wavevector components $h$ and $k_{0}$ is

$$
k_{0}=\left(h^{2}-\varepsilon_{m} \mu_{m} \frac{\omega^{2}}{c^{2}}\right)^{1 / 2} .
$$

For the fields in the region $0<x<d$ we use the form

$$
\begin{aligned}
& \vec{H}^{(2)}=\hat{a}_{y}\left(B \mathrm{e}^{-k x}+C \mathrm{e}^{k x}\right) \mathrm{e}^{\mathrm{i} h z} \\
& \vec{E}^{(2)}=\frac{c}{\omega \varepsilon}\left[B\left(h \hat{a}_{x}-\mathrm{i} k \hat{a}_{z}\right) \mathrm{e}^{-k x}+C\left(h \hat{a}_{x}+\mathrm{i} k \hat{a}_{z}\right) \mathrm{e}^{k x}\right] \mathrm{e}^{\mathrm{i} h z}
\end{aligned}
$$

where

$$
k=\left(h^{2}-\varepsilon(\omega) \mu(\omega) \frac{\omega^{2}}{c^{2}}\right)^{1 / 2}
$$

In the region $x>d$ the fields are written in the form

$$
\begin{aligned}
\vec{H}^{(3)} & =\hat{a}_{y} D \mathrm{e}^{-k_{0} x} \mathrm{e}^{\mathrm{i} h z} \\
\vec{E}^{(3)} & =\frac{c}{\omega \varepsilon_{m}} D\left(h \hat{a}_{x}-\mathrm{i} k_{0} \hat{a}_{z}\right) \mathrm{e}^{-k_{0} x} \mathrm{e}^{\mathrm{i} h z} .
\end{aligned}
$$

The boundary conditions at $x=0$ and $x=d$ require the tangential components of $\vec{E}$ and $\vec{H}$ to be continuous. This yields a system of four homogeneous equations for the coefficients A, B, C and D. Equating the determinant of this system to zero yields the surface polariton dispersion relations

$$
\begin{aligned}
\varepsilon(\omega) & =-\varepsilon_{m} \frac{k}{k_{0}} \operatorname{coth}(k d / 2) \\
\varepsilon(\omega) & =-\varepsilon_{m} \frac{k}{k_{0}} \tanh (k d / 2)
\end{aligned}
$$




\section{2. s polarization}

In this case the fields have the form

$$
\begin{aligned}
& \vec{E}^{(1)}=\hat{a}_{y} A \mathrm{e}^{k_{0} x} \mathrm{e}^{\mathrm{i} h z} \\
& \vec{H}^{(1)}=-\frac{c}{\omega \mu_{m}} A\left(h \hat{a}_{x}+\mathrm{i} k_{0} \hat{a}_{z}\right) \mathrm{e}^{k_{0}} \mathrm{e}^{\mathrm{i} h z}
\end{aligned}
$$

in the region $x<0$, and

$$
\begin{aligned}
& \vec{E}^{(2)}=\hat{a}_{y}\left(B \mathrm{e}^{-k x}+C \mathrm{e}^{k x}\right) \mathrm{e}^{\mathrm{i} h z} \\
& \vec{H}^{(2)}=-\frac{c}{\omega \mu(\omega)}\left[B\left(h \hat{a}_{x}-\mathrm{i} k \hat{a}_{z}\right) \mathrm{e}^{-k x}+C\left(h \hat{a}_{x}+\mathrm{i} k \hat{a}_{z}\right) \mathrm{e}^{k x}\right] \mathrm{e}^{\mathrm{i} h z}
\end{aligned}
$$

in the region $0<x<d$, and

$$
\begin{aligned}
\vec{E}^{(3)} & =\hat{a}_{y} D \mathrm{e}^{-k_{0} x} \mathrm{e}^{\mathrm{i} h z} \\
\vec{H}^{(3)} & =-\frac{c}{\omega \mu_{m}} D\left(h \hat{a}_{x}-\mathrm{i} k_{0} \hat{a}_{z}\right) \mathrm{e}^{-k_{0} x} \mathrm{e}^{\mathrm{i} h z}
\end{aligned}
$$

for $x>d$.

In this case the boundary conditions yield the dispersion relations

$$
\begin{aligned}
& \mu(\omega)=-\mu_{m} \frac{k}{k_{0}} \tanh \left(\frac{k d}{2}\right) \\
& \mu(\omega)=-\mu_{m} \frac{k}{k_{0}} \operatorname{coth}\left(\frac{k d}{2}\right) .
\end{aligned}
$$

\subsection{Numerical results}

Whereas for a semi-infinite medium there exist two surface polariton branches for the $p$ polarization and one branch for the $s$ polarization [7], for the slab the number of branches is doubled. Thus, there exist four surface polariton branches for the $p$ polarization and two for the $s$ polarization. This naturally results from the interaction between the modes which are localized near the two surfaces of the slab. When the limit of a very thick slab is taken, the corresponding pairs of branches become degenerate. These trends will be demonstrated by numerical calculation of the dispersion curves (11), (12), (19) and (20). Our interest here is in surface polaritons, for which the field amplitudes decay exponentially as one moves away from either interface to either direction. This requires both $k_{0}$ and $k$ to be real, and from (5) and (8) it follows that

$$
h^{2}>\varepsilon_{m} \mu_{m}(\omega / c)^{2}
$$

and

$$
h^{2}>\varepsilon(\omega) \mu(\omega)(\omega / c)^{2} .
$$

These two inequalities define two regions in the $(h, \omega)$ plane, in which surface polaritons can exist (figure 1): region $S_{1}$, to the right of line (i) and below line (ii), and region $S_{2}$, to the right of line (i) and above curve (iii). In the numerical calculations, the following values, in the same range as the experimental ones [1], are employed for the parameters appearing in (1) and (2): $\omega_{p}=10 \mathrm{GHz}, \omega_{0}=4 \mathrm{GHz}, F=0.56$. In this case, the frequency range in which both $\varepsilon(\omega)$ and $\mu(\omega)$ are negative extends from $\omega_{0}$ up to $6 \mathrm{GHz}$. For the external medium we assume that $\varepsilon_{m}=1$ and $\mu_{m}=1$. 


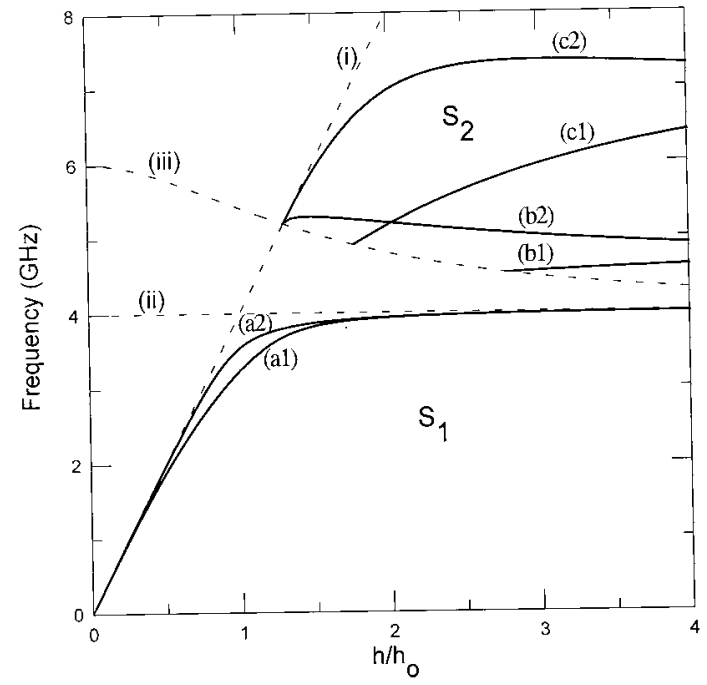

Figure 1. Surface polariton dispersion curves for a slab of thickness $h_{0} d=0.5$. Line (i) is $h=\sqrt{\varepsilon_{m}} \omega / c$, line (ii) is $\omega=\omega_{0}$, and curve (iii) is $h=\sqrt{\varepsilon(\omega) \mu(\omega)} \omega / c . \mathrm{S}_{1}$ and $\mathrm{S}_{2}$ denote the two regions in which surface polaritons can exist. The surface polariton branches (a1), (a2), (c1) and (c2) are for $p$ polarization and branches (b1) and (b2) are for s polarization.

The surface polariton dispersion curves, obtained by solving equations (11), (12), (19) and (20) are shown in figure 1 , for slab thickness given by $h_{0} d=0.5$, where $h_{0}=\omega_{0} / c$. There exist the following three pairs of branches: (a1) and (a2), which are $p$ polarized, and occur in the region $\omega<\omega_{0}$. In this region $\mu(\omega)$ is positive and $\varepsilon(\omega)$ is negative, i.e., the slab material is not left-handed. Branches (b1) and (b2) are $s$ polarized, and occur in the frequency range in which the slab material is left-handed. Branches (c1) and (c2) are $p$ polarized, and each of them crosses over from the region of left-handed behaviour into that of right-handed one.

When the slab thickness increases, the separation between the members of each pair decreases, as demonstrated in figure 2, which was calculated for a thickness of $h_{0} d=1$.

\section{ATR spectra}

The evanescent fields characterizing the surface polaritons do not interact with an incoming electromagnetic plane wave. In order to study the surface polaritons, the attenuated total reflection (ATR) technique can be employed $[8,9]$. The ATR geometry which we consider is shown in figure 3 . The radiation is incident from the right-hand side, at an angle of incidence $\theta$, in a medium having a dielectric constant $\varepsilon_{m}^{\prime}$. This medium is separated from the left-handed slab by a film of thickness $a$, and dielectric constant $\varepsilon_{m}$. The medium on the left-hand side of the slab also has a dielectric constant $\varepsilon_{m}$. We assume that $\mu_{m}=1$ and $\mu_{m}^{\prime}=1$. If the angle of incidence is large enough, so that $\sqrt{\varepsilon_{m}^{\prime}} \sin \theta>\sqrt{\varepsilon_{m}}$, the electromagnetic field will emerge from the first medium only as an evanescent wave, which can interact with the evanescent surface polaritons. We have calculated the reflectivity of the left-handed slab in the configuration of figure 3, using classical electromagnetic theory. For this calculation, a damping parameter $\gamma=0.03$ has been added to both $\varepsilon(\omega)$ and $\mu(\omega)$. As long as the damping is small, the choice of the value of $\gamma$ does not influence the location of the peaks appearing in the ATR spectra. The value employed here enables us to compare our results with the analogous spectra calculated 


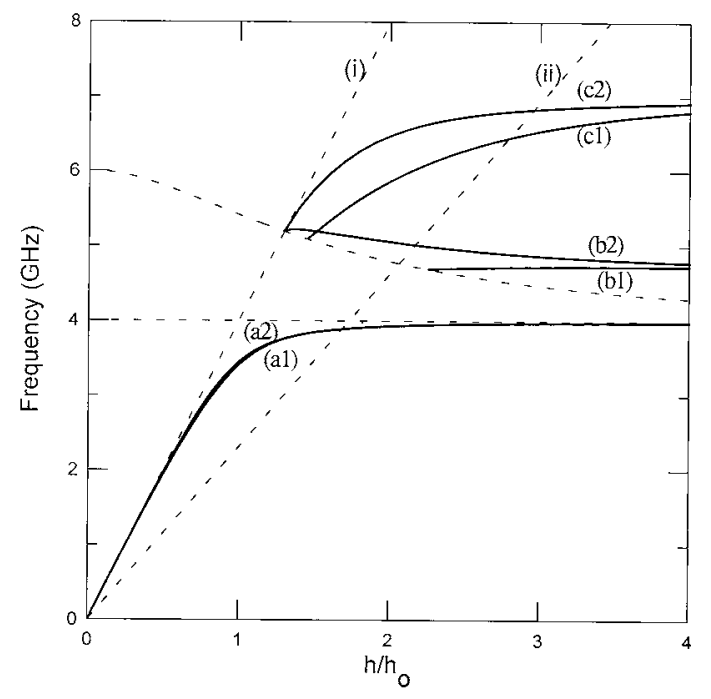

Figure 2. Surface polariton dispersion curves for a slab of thickness $h_{0} d=1.0$. Line (i) is $h=\sqrt{\varepsilon_{m}} \omega / c$, line (ii) is $h=\sqrt{\varepsilon_{m}^{\prime}} \omega / c$. The surface polariton branches (a1), (a2), (c1) and (c2) are for $p$ polarization and branches (b1) and (b2) are for $s$ polarization.

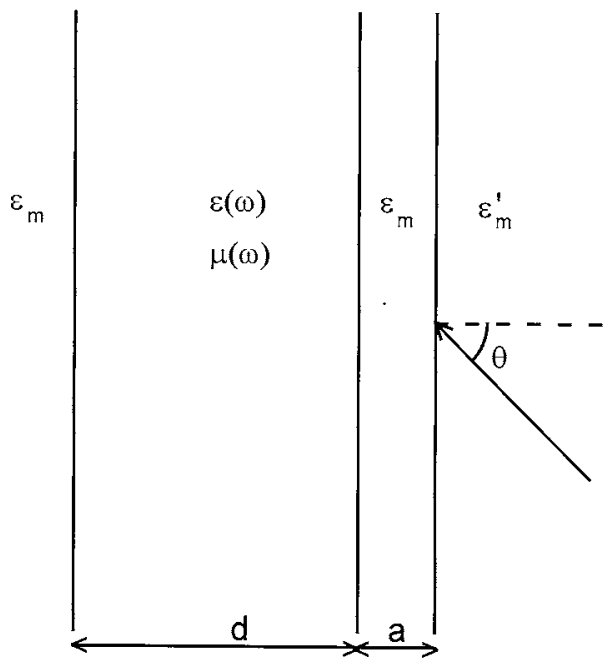

Figure 3. Geometry of ATR experiment.

for a semi-infinite left-handed medium [7].

For the case of $p$ polarization, the ATR spectrum calculated for a thin film, with $h_{0} d=0.5$, is shown in figure 4 . The three peaks denoted $\mathrm{S}$ are due to surface polaritons. The two lowest peaks, at $3.09 \mathrm{GHz}$ and $3.66 \mathrm{GHz}$, are due to branches (a1) and (a2) of figure 1, and the high-frequency peak, at $7.13 \mathrm{GHz}$, is due to the (c2) branch. The peak denoted B lies outside the frequency range of the surface polariton branches, and is due to the excitation of a nonradiative bulk polariton, i.e., a polariton that has a sinusoidal behaviour in the $x$ direction inside the slab, and an exponential decay outside the slab [10]. When the slab thickness is increased to $h_{0} d=1$, the spectrum shown in figure 5 is obtained. Now only one low-frequency surface 


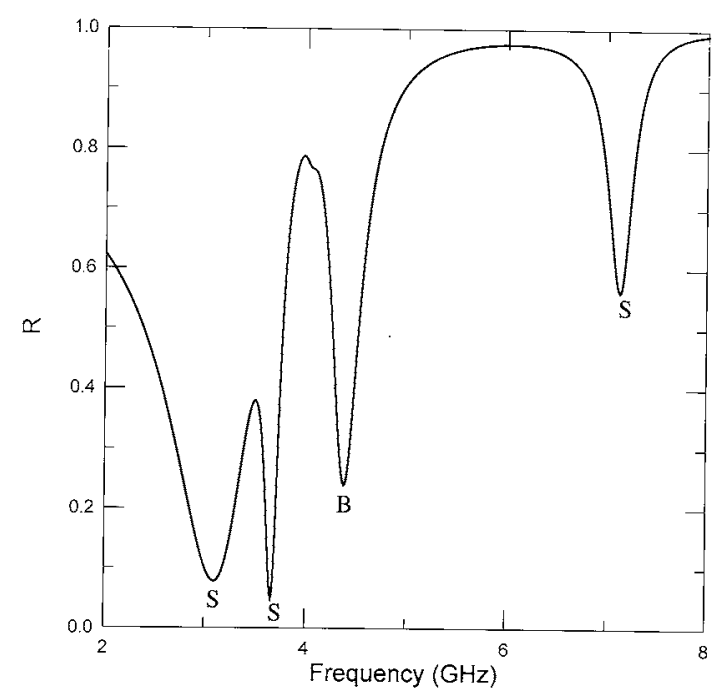

Figure 4. Calculated reflectivity for $p$ polarization. The parameters used in this calculation are $h_{0} d=0.5, \varepsilon_{m}=1, \varepsilon_{m}^{\prime}=3, a=2 \mathrm{~cm}, \theta=45^{\circ}$.

mode peak appears, at $3.49 \mathrm{GHz}$, because branches (a1) and (a2) of figure 2 are practically degenerate. The high-frequency branches (c1) and (c2) of figure 2 now manifest themselves in two high-frequency peaks, at $5.39 \mathrm{GHz}$ and $6.43 \mathrm{GHz}$. For comparison, the dashed curve of figure 5 shows the corresponding reflectivity of a semi-infinite medium [7]. It can be seen that the low-frequency peak of the slab is already located at the position of the corresponding peak of a semi-infinite medium, but the two high-frequency peaks have not yet converged to the semi-infinite medium value.

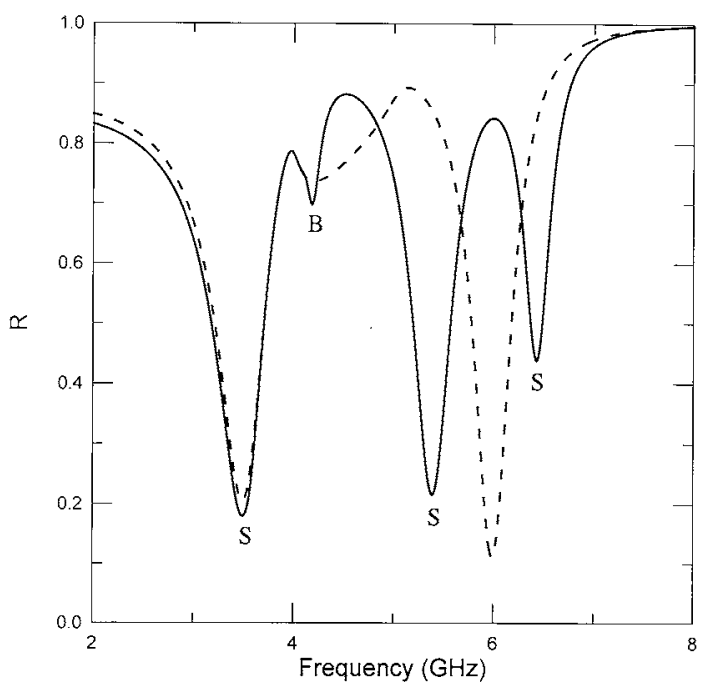

Figure 5. Full curve-calculated reflectivity for $p$ polarization. The parameters used in this calculation are $h_{0} d=1.0, \varepsilon_{m}=1, \varepsilon_{m}^{\prime}=3, a=2 \mathrm{~cm}, \theta=45^{\circ}$. The dashed curve shows the corresponding reflectivity of a semi-infinite medium. 


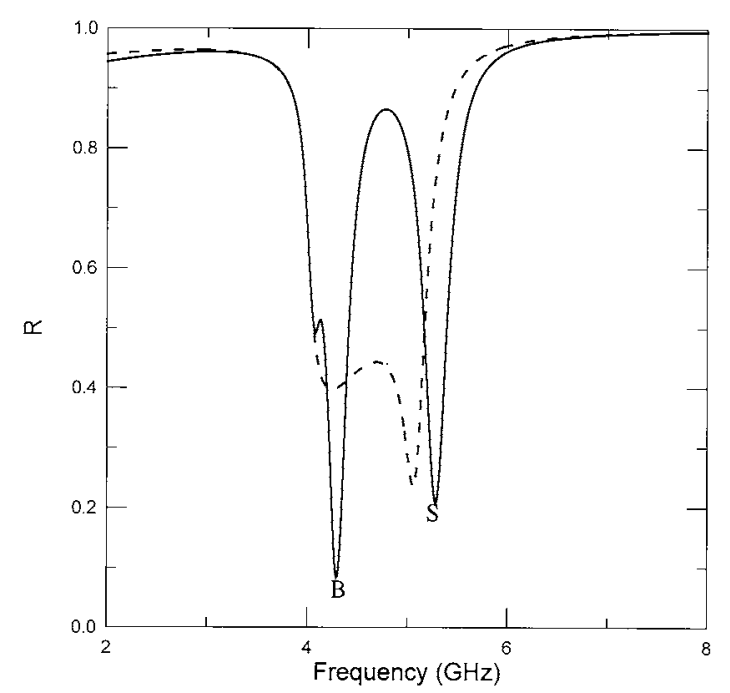

Figure 6. Full curve-calculated reflectivity for $s$ polarization. The parameters used in this calculation are $h_{0} d=0.5, \varepsilon_{m}=1, \varepsilon_{m}^{\prime}=3, a=1 \mathrm{~cm}, \theta=45^{\circ}$. The dashed curve shows the corresponding reflectivity of a semi-infinite medium.

An ATR spectrum calculated for the case of $s$ polarization is shown in figure 6. In addition to the bulk mode absorption, denoted $\mathrm{B}$, only one surface mode peak, denoted $\mathrm{S}$, is observed. This is due to the surface polariton branch (b2) of figure 1. The region in which the surface polariton branches can be observed by the ATR method is bounded by lines (i) and (ii) of figure 2, defined by $h=\sqrt{\varepsilon_{m}} \omega / c$ and $h=\sqrt{\varepsilon_{m}^{\prime}} \omega / c$, respectively. Since branch (b1) lies outside this region, it cannot be observed in the ATR measurement. For comparison, the corresponding spectrum of a semi-infinite medium [7] is also shown in figure 6.

\section{Discussion}

We have derived the dispersion curves of the polaritons that are localized near the two surfaces of a slab, which is characterized by dispersive permittivity and permeability, and which is lefthanded over a frequency band. The number of surface polariton branches is twice the number of the corresponding branches of a semi-infinite medium. This is due to the interaction between the modes that are localized on either side of the slab, which causes a split up. The two lowest frequency surface polariton branches are $p$ polarized, and are located in the frequency range below the magnetic resonance frequency $\omega_{0}$. In this frequency range the material is not lefthanded. The next two branches are $s$ polarized, and are located above $\omega_{0}$, completely within the frequency region in which the material is left-handed. The two highest surface polariton branches are again $p$ polarized. They are partly within the frequency range of left-handness and partly outside it, at higher frequencies.

In conventional dielectric or metallic material slabs, with dispersive $\varepsilon(\omega)$, but no magnetic dispersion, surface polaritons exist only in the stop band, in which the permittivity is negative. In magnetic material slabs, with dispersive $\mu(\omega)$, but no dielectric dispersion, again surface polaritons exist only in the stop band, in which the permeability is negative. Thus a lefthanded material slab has the unusual property of possessing surface localized modes within the frequency pass band (in which both the permittivity and the permeability are negative). This property was demonstrated by calculations based on a specific choice of the permittivity 
and permeability parameters, but should have a wider validity.

In ATR spectra the surface polaritons manifest themselves as sharp reflectivity minima. Although there is no energy flow through the slab to the other direction, due to the evanescent nature of the fields, at the surface polariton frequencies there also occur peaks of the field amplitudes near the distant surface of the slab. Thus, these polaritons can be utilized in the design of lenses based on left-handed materials [3].

\section{References}

[1] Smith D R, Padilla W J, Vier D C, Nemat-Nasser S C and Schultz S 2000 Phys. Rev. Lett. 844184

[2] Veselago V G 1968 Sov. Phys. Uspekhi 10509

[3] Pendry J B 2000 Phys. Rev. Lett. 853966

[4] Pendry J B, Holden A J, Robbins D J and Stewart W J 1998 J. Phys.: Condens. Matter 104785

[5] Pendry J B, Holden A J, Robbins D J and Stewart W J 1999 IEEE Trans. Microwave Theory Tech. 472075

[6] Smith D R, Vier D C, Kroll N and Schultz S 2000 Appl. Phys. Lett. 772246

[7] Ruppin R 2000 Phys. Lett. A 27761

[8] Otto A 1969 Z. Physik 219227

[9] Ruppin R 1970 Solid State Commun. 81129

[10] Kliewer K L and Fuchs R 1966 Phys. Rev. 144495 